\title{
A post-recurrence survival-predicting indicator for cervical cancer from the analysis of 165 patients who developed recurrence
}

\author{
KOSUKE YOSHIDA ${ }^{1}$, HIROAKI KAJIYAMA ${ }^{1}$, FUMI UTSUMI $^{1}$, KAORU NIIMI $^{1}$, \\ JUN SAKATA ${ }^{1}$, SHIRO SUZUKI ${ }^{1}$, KIYOSUMI SHIBATA ${ }^{2}$ and FUMITAKA KIKKAWA ${ }^{1}$ \\ ${ }^{1}$ Department of Obstetrics and Gynecology, Graduate School of Medicine, Nagoya University, Nagoya 466-8550; \\ ${ }^{2}$ Department of Obstetrics and Gynecology, Banbuntane Hotokukai Hospital, Nagoya 454-8509, Japan
}

Received June 5, 2017; Accepted September 28, 2017

DOI: $10.3892 /$ mco.2017.1530

\begin{abstract}
The aim of the present study was to estimate the post-recurrence survival (PRS) of patients with relapsed uterine cervical cancer (RUCC). In addition, clinicopathological indicators that influenced PRS were investigated. Between 1998 and 2014, of 740 patients with cervical cancer, 165 patients experienced recurrence (recurrence rate, 22.3\%), and 83 patients succumbed to the disease within a median follow-up of 34.3 months. A total of 151 stage Ib-IV patients who experienced recurrence after initial treatment for cervical cancer at our institute were analyzed. Uni- and multivariate analyses were performed using the Kaplan Meier method, and Cox regression model. The median age was 55 years (range, $20-88$ years). In all, 80 patients succumbed to the disease. The median PRS time of all the patients was 28.4 months. The 1-, 3-, and 5-year PRS rates of patients were 75.1, 41.9, and $32.1 \%$, respectively. In addition, the median survival period in patients who had received surgery as an initial treatment was significantly longer compared with that in patients who had not previously undergone surgery (36.7 vs. 23.3 months, respectively; $\mathrm{P}=0.0338$ ). Following the univariate analysis, the median PRS in patients with in- and out-field recurrence was 12.6, and 45.9 months, respectively $(\mathrm{P}<0.0001)$. Furthermore, in the multivariable analysis, the recurrence site was a significant prognostic indicator of PRS [(In-field vs. Out-field); hazard ratio, 2.848; 95\% confidence interval, 1.707-4.738; $\mathrm{P}<0.0001]$. The long-term clinical outcome of patients with RUCC was poor. In particular, the in-field recurrence was identified to be
\end{abstract}

Correspondence to: Dr Hiroaki Kajiyama, Department of Obstetrics and Gynecology, Graduate School of Medicine, Nagoya University, Tsuruma-cho 65, Showa-ku, Nagoya 466-8550, Japan E-mail: kajiyama@med.nagoya-u.ac.jp

Abbreviations: RUCC, recurrent uterine cervical carcinoma; PRS, post-recurrence survival

Key words: cervical cancer, post-recurrence survival, in-field recurrence, salvage treatment associated with poor post-recurrence oncological outcome in patients with RUCC.

\section{Introduction}

Cervical cancer is one of the most common malignancies in females worldwide. There were an estimated 527,600 new cervical cancer cases and 265,700 deaths in 2012 according to cancer statistics (1), despite the mortality decreasing due to widespread screening programs and the increased use of the human papillomavirus vaccine in Western countries.

Although radical surgery and concurrent chemoradiotherapy (CCRT) have been established as the standard therapy for patients with localized cervical cancer and locally advanced carcinoma, one third of patients experience recurrence and the effect of treatment for recurrence remains far from satisfactory, resulting in a 5-year survival rate after recurrence of less than $5 \%(2,3)$. Furthermore, little is known about the factors indicating the prognosis after recurrence. Tokunaga et al analyzed the effect of chemotherapy on patients with recurrent uterine cervical cancer (RUCC) after CCRT, and revealed that the overall response and survival rates did not differ significantly according to the recurrence site, post-CCRT interval, or chemotherapy regimen (3). Additionally, some studies reported the survival benefit of surgical intervention for recurrent or persistent uterine and cervical malignancies (4). Treatments for RUCC mainly depend on the previous treatments and sites of recurrence (5), and the prognosis may also be affected by the treatment for recurrence. The factors that affect the prognosis of RUCC patients remain controversial.

The aim of this study was to evaluate the long-term clinical outcome and elucidate the prognostic factors in patients with RUCC.

\section{Patients and methods}

Patients. We retrospectively reviewed all the records of 740 patients with uterine cervical cancer who were initially treated in our hospital from January 1998 to December 2014. This study was approved by the Ethics Committee of Nagoya University. One hundred and sixty-five patients experienced recurrence. Their clinical data including age, pathological or clinical stage, histological subtype, initial treatment, recur- 
rence site, date of recurrence diagnosis, and outcomes were collected. Nine patients with insufficient information and five patients with stage IA were excluded, and finally 151 patients were included in the present analysis.

Methods. Primary treatments for each patient were determined by several gynecologic oncologists in our hospital depending on their age, performance status (PS), and International Federation of Gynecology and Obstetrics (FIGO) stage. For example, early-stage patients with good PS were indicated for radical hysterectomy with or without adjuvant CCRT. Patients who were contraindicated for radical surgery were mostly treated with primary CCRT, chemotherapy, or radiotherapy (RT) alone. Cisplatin (70 mg/m², on day 1) and 5-fluorouracil (700 mg/m $\mathrm{m}^{2}, 24-\mathrm{h}$ continuous intravenous infusion, on days 1-4) combination chemotherapy was usually administered as the initial chemotherapy. When creatinine clearance was $<60 \mathrm{ml} / \mathrm{min}$, nedaplatin or carboplatin was considered instead of cisplatin. RT involved a combination of external beam radiotherapy (ERBT) and intra-cavity brachytherapy (ICBT). ERBT was performed at 1.8 Gy once per day (total dose of 50.4 Gy in 28 fractions). ICBT was performed during external beam radiation therapy using a remote after loading the system with a Co 60 source. The total dose to point A (a reference location $2 \mathrm{~cm}$ lateral and $2 \mathrm{~cm}$ superior to the cervical os) was 18-24 Gy. The radiation field extended from the space between L4 and L5 to the base of the obturator foramen.

At the end of the treatment, all the patients underwent a strict follow-up consisting of clinical checkups such as a pelvic examination, ultrasonographic scan, serological tumor marker evaluation, and periodic computed tomographic scan/positron emission tomography. Patients with radiologic recurrence were defined as those who were found to have tumor recurrence based on computed tomography, magnetic resonance imaging, positron emission tomography, or ultrasound. Images were viewed by at least a gynecologic oncologist and a radiologist. Post-recurrence survival (PRS) was calculated from the date of recurrence diagnosis to the date of death or that of the last follow-up. Post-recurrence treatments such as surgical intervention, radiotherapy, and/or chemotherapy were performed depending on the individual cases. Most of the patients received several courses of chemotherapy such as paclitaxel plus carboplatin, irinotecan, or nedaplatin. Some patients underwent secondary radical surgery including pelvic exenteration, and some patients received lobectomy for solitary pulmonary metastasis. Patients who had PAN metastasis or pelvic recurrence without a history of irradiation received radiotherapy. In-field recurrence was defined as a recurrence where previous radiotherapy had been conducted.

Statistical analysis. Statistical analysis was performed using JMP Pro 11. Survival analysis was based on the Kaplan-Meier method, and survival curves were compared using the log-rank test. Multivariable analysis was performed with the Cox proportional hazard model to evaluate independent factors possibly affecting survival. $\mathrm{P}<0.05$ was considered significant.

\section{Results}

Patient characteristics. Of the 740 cervical cancer patients, 165 patients experienced recurrence (recurrence rate: $22.3 \%$ ), and 83 patients died within a median follow-up of 34.3 months. Of the 165 recurrence patients, 9 were excluded because sufficient information was not available. In addition, five stage IA patients who experienced recurrence were also excluded. As a consequence, 151 stage IB-IVB patients who experienced recurrence were included in this study. Clinicopathologic characteristics of patients are described in Table I. The median age was 55 years (range, 20-88) years. The most common clinical stage according to the FIGO staging system was Stage II (43.7\%). Squamous cell carcinomas (SCC) were the most frequently observed histological type (63.6\%) followed by adenocarcinomas (AC) $(29.1 \%)$. As an initial treatment, 70 (46.3\%) patients underwent radical surgery with or without adjuvant therapy, while $81(53.7 \%)$ patients were treated with primary CCRT or primary RT. Solitary recurrences in the pelvis were observed in 54 patients $(35.8 \%)$, and recurrences in para-aortic lymph nodes (PAN) regardless of pelvic recurrence were diagnosed in 32 patients $(21.2 \%)$. Sixty-three (41.7\%) patients experienced recurrence in distant or mixed regions. Generally, in- or out-field recurrence was observed in 43 and 88 patients, respectively.

Kaplan-Meier method. The median PRS period was 28.4 months (range, 0-154.7 months), and the 1-, 3-, and 5-year PRS rates of patients were $75.1,41.9$, and $32.1 \%$, respectively (Fig. 1). There was no difference in PRS of patients between the two age categories ( $<55$ vs. $\geq 55$ years) (Fig. 2 ). In addition, the median survival period in patients who had received surgery as an initial treatment was significantly longer compared with that in patients who had never undergone surgery (36.7 vs. 23.3 months, respectively, $\mathrm{P}=0.0338$ ) (Fig. 3). Furthermore, the median survival period in patients who had experienced in-field recurrence was significantly shorter compared with that in patients who had experienced out-field or radiotherapy-free recurrence (in-field vs. out-field: 12.6 vs. 45.9 months, respectively, $\mathrm{P}<0.0001$ ) (Fig. 4).

Univartiate and multivariate analyses. Uni- and multivariate analyses of PRS according to the patients' characteristics and recurrent features were performed. Age ( $\leq 55$ vs. $>55)$, FIGO stage (I vs. II/III/IV), histological type (SCC vs. non-SCC), initial treatment (surgery vs. CCRT/RT), and recurrence site (in-field vs. out-field vs. RT-free) were analyzed. The results of multivariate analysis are shown in Table II. No significant differences were found in the age, FIGO Stage, histological type, or modality of initial treatment. However, the in-field recurrence had an impact on PRS after the multivariate analysis [in-field vs. out-field: HR (95\% CI): 2.848 (1.707-4.738), $\mathrm{P}<0.0001]$.

\section{Discussion}

The present study involved a large-scale retrospective analysis to evaluate the post-recurrence clinical outcomes of RUCC patients. Although much supporting evidence of initial treatment including CCRT has been established and the survival rate has been improved $(6,7)$, little is known regarding the optimal treatment for patients with RUCC, and the prognosis of RUCC patients is poor. In this study, we analyzed clinical outcomes of patients with RUCC in our institute and evaluated 
Table I. Patient characteristics.

\begin{tabular}{lrr}
\hline Characteristic & $\mathrm{N}$ & $\%$ \\
\hline Total & 151 & \\
Median age & 55 & \\
(Range) & $20-88$ & \\
Stage & & 22.5 \\
IB & 34 & 43.7 \\
II & 66 & 9.9 \\
III & 15 & 23.8 \\
IV & 36 & \\
Histological type & & 63.6 \\
SCC & 96 & 29.1 \\
AC & 44 & 4.6 \\
AS & 7 & 2.6 \\
Small cell & 4 & 1.3 \\
Initial treatment & & 33.1 \\
NAC-surgery & 2 & 11.9 \\
NACCRT-surgery & 50 & 43.7 \\
Surgery & 18 & 9.9 \\
Primary CCRT & 66 & \\
Primary RT & 15 & \\
Recurrence site & & \\
Out-field & & \\
In-field & & \\
w/o RT & & \\
\hline
\end{tabular}

SCC, squamous cell carcinoma; AC, adenocarcinoma; AS, adenosquamous carcinoma; NAC, neoadjuvant chemotherapy plus surgery; NACCRT, neoaduvant chemoradiotherapy plus surgery; CCRT, chemoradiotherapy alone; RT, radiotherapy alone.

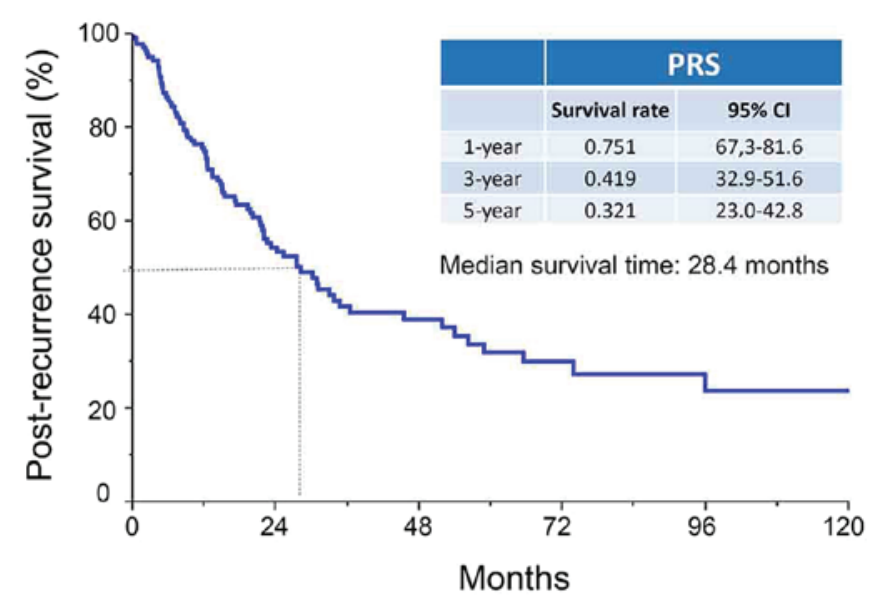

Figure 1. Kaplan-Meier-estimated post-recurrence survival (PRS) of all patients enrolled. The 5-year PRS was $32.1 \%$ and median survival time (MST) was 28.4 months.

prognostic factors concerning PRS instead of overall survival (OS) or progression-free survival (PFS). The recurrence rate of all the patients was $22.3 \%$, which was consistent with

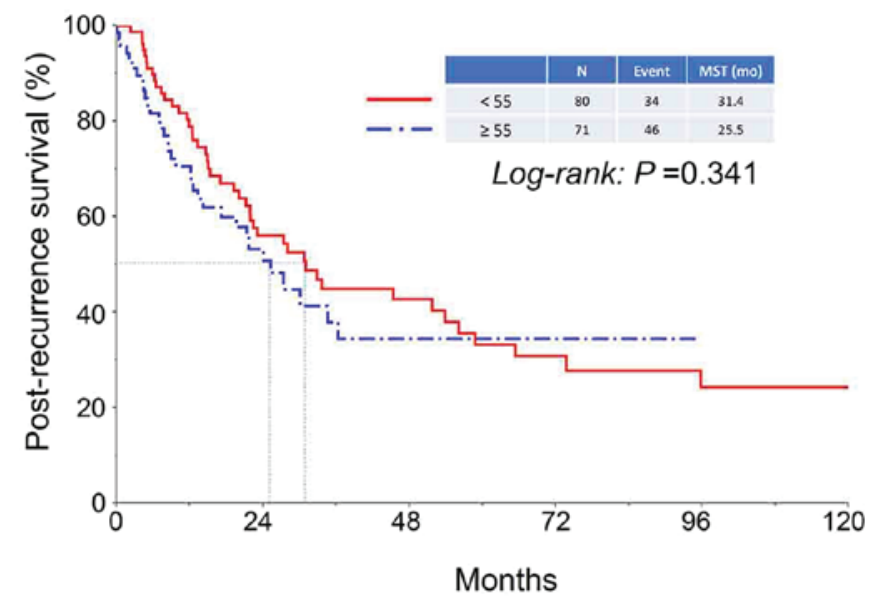

Figure 2. Kaplan-Meier-estimated PRS of patients on stratifying by age ( $\leq 55$ vs. $>55$ years). Log-Rank: $\mathrm{P}=0.341$.

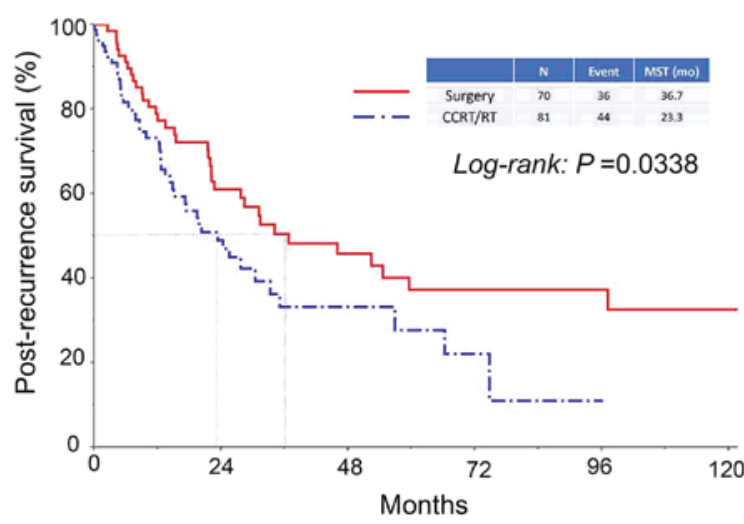

Figure 3. Kaplan-Meier-estimated PRS of patients on stratifying by the type of treatment (patients on whom surgery had been performed as initial treatment or had never been performed). Log-Rank: $\mathrm{P}=0.0338$.

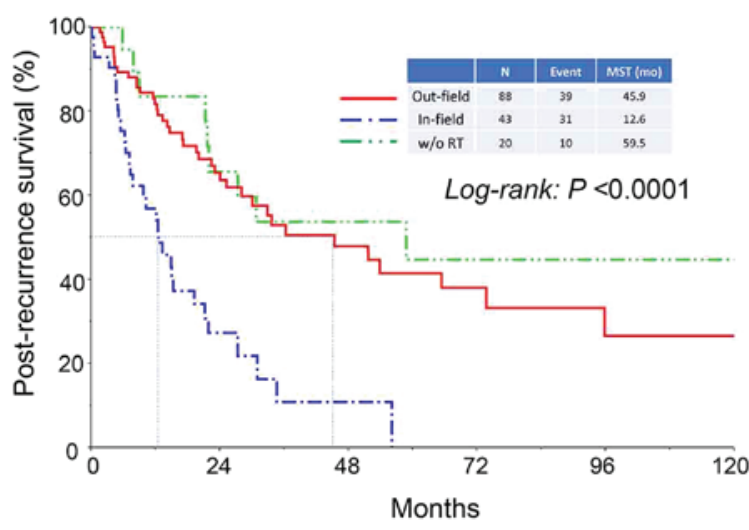

Figure 4. Kaplan-Meier-estimated PRS of patients on stratifying by recurrence site. The median survival period in patients who had experienced in-field recurrence was significantly shorter compared with that in patients who had experienced out-field or radiotherapy-free recurrence (in-field vs. out-field: 12.6 vs. 45.9 months, respectively). $\mathrm{P}<0.0001$.

previous reports (8-12). As expected, patients who experienced recurrence had an extremely poor prognosis (median PRS of 28.4 months and 5-year PRS rate of 32.1\%). We identified two 
Table II. Uni- and multivariable analyses of clinicopathological parameters in relation to post-recurrence survival of patients enrolled. ${ }^{\mathrm{a}}$

\begin{tabular}{|c|c|c|c|c|c|c|}
\hline \multirow[b]{2}{*}{ Characteristic } & \multicolumn{3}{|c|}{ Univariable analysis } & \multicolumn{3}{|c|}{ Multivariable analysis } \\
\hline & Hazard ratio & $95 \% \mathrm{CI}$ & P-value & Hazard ratio & $95 \% \mathrm{CI}$ & P-value \\
\hline \multicolumn{7}{|l|}{ Age } \\
\hline$<55$ & Referent & & & Referent & & \\
\hline$\geq 55$ & 1.245 & $0.511-1.270$ & 0.345 & 1.076 & $0.650-1.776$ & 0.772 \\
\hline \multicolumn{7}{|l|}{ FIGO Stage } \\
\hline I & Referent & & & Referent & & \\
\hline II / III / IV & 1.367 & $0.824-2.385$ & 0.232 & 1.101 & $0.603-2.077$ & 0.758 \\
\hline \multicolumn{7}{|c|}{ Histological type } \\
\hline $\mathrm{SCC}$ & Referent & & & Referent & & \\
\hline Non-SCC & 0.890 & $0.569-1.412$ & 0.616 & 1.202 & $0.725-1.970$ & 0.469 \\
\hline \multicolumn{7}{|c|}{ Initial treatment } \\
\hline Surgery & Referent & & & Referent & & \\
\hline CCRT/RT & 1.627 & $1.036-2.578$ & 0.0344 & 1.259 & $0.712-2.255$ & 0.429 \\
\hline \multicolumn{7}{|l|}{ Recurrence site } \\
\hline Out-field & Referent & & & Referent & & \\
\hline In-field & 3.043 & $1.856-4.962$ & $<0.0001$ & 2.848 & $1.707-4.738$ & $<0.0001$ \\
\hline w/o RT & 0.766 & $0.347-1.515$ & 0.461 & 0.854 & $0.359-1.877$ & 0.705 \\
\hline
\end{tabular}

${ }^{a}$ Cox hazard model. FIGO, International Federation of Gynecology and Obstetrics; SCC, squamous cell carcinoma; CCRT, chemoradiotherapy; RT, radiotherapy alone; w/o RT, recurrence without prior radiotherapy; CI, confidence interval.

prognostic factors affecting PRS. The in-field recurrence and ineligibility for primary radical surgery were significantly correlated with a poor prognosis.

We showed the significant impact of initial surgery on PRS. According to the findings of Rungruang et al, patients with stage IB2 cervical cancer, and patients who received primary surgery showed a longer survival than those who received primary radiotherapy regardless of chemotherapy. Although the details of chemotherapy were not clear, it was a large-scale study using the Surveillance, Epidemiology, and End Results (SEER) database (13). In addition, Derks et al suggested that radical surgery may be a good treatment option for patients with stage IB2/IIA2 cervical cancer because patients who received surgery-based treatment showed relatively longer survival (14). Therefore, surgical intervention is considered a favorable treatment for local cervical carcinoma. Furthermore, some reports showed an impact of the surgical procedure on advanced or recurrent cervical cancer $(8,15)$.

We showed that pelvic recurrence was significantly correlated with a poor prognosis. Legge et al reported that the median survival of patients with visceral (e.g., lungs, bones, liver, brain) or lymph nodal metastatic relapse was significantly better compared with that in patients with pelvic recurrence (8). By contrast, other reports concluded that pelvic recurrence could be salvaged with CCRT in patients who were not initially treated with radiation therapy, and that central pelvic recurrence might be salvaged with pelvic exenteration (16-18). Additionally, it has been considered that vaginal recurrence after radical surgery was related to a good prognosis (12), while distant metastases have been considered incurable except for isolated pulmonary metastasis $(16,17)$. Concerning PAN metastasis, the prognosis of those patients may not be worse than expected because CCRT contributed to longer survival $(5,12,19)$. A previous report suggested that there was no difference in the survival rate between patients with recurrence in a previously irradiated field and those with recurrence in an extra-irradiated field (3). However, the unfavorable outcome of in-field recurrence in our study is due to the fact that tumor recurred in the irradiated field may have a more aggressive biological behavior with more radio- and/or chemo-resistant hallmark. In the actual clinical situation, treatment options for such tumors are extremely limited.

Finally, considering our two negative prognostic factors, local control of carcinoma by radical surgery may be important for survival. In addition, another study reported that both OS and PFS were improved with neoadjuvant chemotherapy followed by radical surgery for patients with early-stage or locally-advanced cervical cancer (20).

There are several limitations to the present study because of the retrospective nature based on our clinical records. First, detailed information including the extent of the disease, performance status, and intraoperative findings was unclear. Second, because our cases were accumulated over a long time, the salvage chemotherapy for RUCC was not necessarily homogenous. Furthermore, information on salvage cytoreductive surgery was lacking. Tumor debulking for solitary mass of RUCC appears to be effective. We hope to verify this in a future study. 
In conclusion, the performance of radical surgery as an initial treatment and out-field/RT-free recurrence are good prognostic factors after the recurrence of cervical cancer. Particularly, in-field recurrence was an independent indicator for poorer post-recurrence survival. Coping with this type of recurrence is major critical issue for the improvement of patient prognosis. If a tumor is limited in the central part of pelvis without dense adhesion to the pelvic wall, we should not hesitate to carry out the pelvic exenteration. However, if we encounter a patient who experience in-field recurrence, with chemoresistance, earlier induction of palliative care needs to be considered rather than continuing chemotherapy. To assess the further appropriateness of in-field recurrence, we would like to accumulate more cases and reconfirm the current results in the future.

\section{References}

1. Torre LA, Bray F, Siegel RL, Ferlay J, Lortet-Tieulent J and Jemal A: Global Cancer Statistics, 2012. CA Cancer J Clin 65: 87-108, 2015.

2. Allemani C, Weir HK, Carreira H, Harewood R, Spika D, Wang XS, Bannon F, Ahn JV, Johnson CJ, Bonaventure A, et al: Global surveillance of cancer survival 1995-2009: Analysis of individual data for $25,676,887$ patients from 279 population-based registries in 67 countries (CONCORD-2). Lancet 385: 977-1010, 2015.

3. Tokunaga H, Nakanishi T, Iwata T, Aoki D, Saito T, Nagase S, Takahashi F, Yaegashi N and Watanabe Y: Effects of chemotherapy on patients with recurrent cervical cancer previously treated with concurrent chemoradiotherapy: A retrospective multicenter survey in Japan. Int J Clin Oncol 20: 561-565, 2015.

4. Andikyan V, Khoury-Collado F, Sonoda Y, Gerst SR, Alektiar KM, Sandhu JS, Bochner BH, Barakat RR, Boland PJ and Chi DS: Extended pelvic resections for recurrent or persistent uterine and cervical malignancies: An update on out of the box surgery. Gynecol Oncol 125: 404-408, 2012.

5. Gadducci A, Tana R, Cosio S and Cionini L: Treatment options in recurrent cervical cancer (Review). Oncol Lett 1: 3-11, 2010.

6. Morris M, Eifel PJ, Lu J, Grigsby PW, Levenback C, Stevens RE, Rotman M, Gershenson DM and Mutch DG: Pelvic radiation with concurrent chemotherapy compared with pelvic and para-aortic radiation for high-risk cervical cancer. N Engl J Med 340: 1137-1143, 1999.

7. Green JA, Kirwan JM, Tierney JF, Symonds P, Fresco L, Collingwood M and Williams CJ: Survival and recurrence after concomitant chemotherapy and radiotherapy for cancer of the uterine cervix: A systematic review and meta-analysis. Lancet 358: 781-786, 2001.

8. Legge F, Chiantera V, Macchia G, Fagotti A, Fanfani F, Ercoli A, Gallotta V, Morganti AG, Valentini V, Scambia G and Ferrandina G: Clinical outcome of recurrent locally advanced cervical cancer (LACC) submitted to primary multimodality therapies. Gynecol Oncol 138: 83-88, 2015
9. Gadducci A, Sartori E, Maggino T, Zola P, Cosio S, Zizioli V, Lapresa M, Piovano E and Landoni F: Pathological response on surgical samples is an independent prognostic variable for patients with Stage Ib2-IIb cervical cancer treated with neoadjuvant chemotherapy and radical hysterectomy: An Italian multicenter retrospective study (CTF Study). Gynecol Oncol 131: 640-644, 2013.

10. Singh AK, Grigsby PW, Rader JS, Mutch DG and Powell MA: Cervix carcinoma, concurrent chemoradiotherapy, and salvage of isolated paraaortic lymph node recurrence. Int J Radiat Oncol Biol Phys 61: 450-455, 2005.

11. Rose PG, Java J, Whitney CW, Stehman FB, Lanciano R, Thomas GM and DiSilvestro PA: Nomograms predicting progression-free survival, overall survival, and pelvic recurrence in locally advanced cervical cancer developed from an analysis of identifiable prognostic factors in patients from NRG Oncology/Gynecologic oncology group randomized trials of chemoradiotherapy. J Clin Oncol 33: 2136-2142, 2015.

12. Qiu JT, Abdullah NA, Chou HH, Lin CT, Jung SM, Wang CC, Chen MY, Huang KG, Chang TC and Lai CH: Outcomes and prognosis of patients with recurrent cervical cancer after radical hysterectomy. Gynecol Oncol 127: 472-477, 2012.

13. Rungruang B, Courtney-Brooks M, Beriwal S, Zorn KK, Richard SD, Olawaiye AB, Krivak TC and Sukumvanich P: Surgery versus radiation therapy for stage IB2 cervical carcinoma: A population-based analysis. Int J Gynecol Cancer 22: 484-489, 2012.

14. Derks M, Biewenga P, van der Velden J, Kenter GG, Stalpers LJA and Buist MR: Results of radical surgery in women with stage IB2/IIA2 cervical cancer. Acta Obstet Gynecol Scand 95: 166-172, 2016.

15. Schmidt AM, Imesch P, Fink D and Egger H: Indications and long-term clinical outcomes in 282 patients with pelvic exenteration for advanced or recurrent cervical cancer. Gynecol Oncol 125: 604-609, 2012.

16. Elit L and Reade CJ: Recommendations for Follow-up care for gynecologic cancer survivors. Obstet Gynecol 126: 1207-1214, 2015.

17. Waggoner SE: Cervical cancer. Lancet 361: 2217-2225, 2003.

18. Mabuchi S, Isohashi F, Yoshioka Y, Temma K, Takeda T, Yamamoto T, Enomoto T, Morishige K, Inoue T and Kimura T: Prognostic factors for survival in patients with recurrent cervical cancer previously treated with radiotherapy. Int J Gynecol Cancer 20: 834-840, 2010.

19. Jang H, Chun M, Cho O, Heo JS, Ryu HS and Chang SJ: Prognostic factors and treatment outcome after radiotherapy in cervical cancer patients with isolated para-aortic lymph node metastases. J Gynecol Oncol 24: 229-235, 2013.

20. Rydzewska L, Tierney J, Vale CL and Symonds PR: Neoadjuvant chemotherapy plus surgery versus surgery for cervical cancer. Cochrane Database Syst Rev 12: CD007406, 2012. 